\title{
Low energy neutral atom imaging on the Moon with the SARA instrument aboard Chandrayaan-1 mission
}

\author{
Anil Bhardwaj ${ }^{1, *}$, Stas Barabash $^{2}$, Yoshifumi Futaana ${ }^{2}$, Yoichi Kazama ${ }^{2}$, \\ Kazushi Asamura ${ }^{3}$, David MCCann ${ }^{2}$, R Sridharan ${ }^{1}$, Mats Holmstrom ${ }^{2,5}$, \\ Peter Wurz ${ }^{4}$ and RickARd LUNDin ${ }^{2}$ \\ ${ }^{1}$ Space Physics Laboratory, Vikram Sarabhai Space Centre, Trivandrum 695 022, India. \\ *e-mail: anil_bhardwaj@vssc.org \\ ${ }^{2}$ Swedish Institute of Space Physics, Box 812, 98128, Kiruna, Sweden. \\ ${ }^{3}$ Japan Aerospace Exploration Agency, Sagamihara, Kanagawa 229-8510, Japan. \\ ${ }^{4}$ Physikalisches Institut, University of Bern, CH-3012 Bern, Switzerland. \\ ${ }^{5}$ Currently at NASA Goddard Space Flight Center, Mail Code 612.2, Greenbelt, MD 20771, USA.
}

This paper reports on the Sub-keV Atom Reflecting Analyzer (SARA) experiment that will be flown on the first Indian lunar mission Chandrayaan-1. The SARA is a low energy neutral atom (LENA) imaging mass spectrometer, which will perform remote sensing of the lunar surface via detection of neutral atoms in the energy range from $10 \mathrm{eV}$ to $3 \mathrm{keV}$ from a $100 \mathrm{~km}$ polar orbit. In this report we present the basic design of the SARA experiment and discuss various scientific issues that will be addressed. The SARA instrument consists of three major subsystems: a LENA sensor (CENA), a solar wind monitor (SWIM), and a digital processing unit (DPU). SARA will be used to image the solar wind-surface interaction to study primarily the surface composition and surface magnetic anomalies and associated mini-magnetospheres. Studies of lunar exosphere sources and space weathering on the Moon will also be attempted. SARA is the first LENA imaging mass spectrometer of its kind to be flown on a space mission. A replica of SARA is planned to fly to Mercury onboard the BepiColombo mission.

\section{Introduction}

A planetary exploration program has been initiated by India with a mission to the Moon the Chandrayaan-1 mission (Bhandari 2004; Thyagarajan and Annadurai 2004; Adimurthy and Ramanan 2004; Goswami 2005). 'Chandrayaan' is a Sanskrit word made by combing two words, Chandra + Yaan, which mean Moon + craft (or vehicle), that is 'lunar spacecraft'. Chandrayaan-1 will be launched in end 2007 and is expected to have a lifetime of 2 years. The spacecraft will be placed in a $100 \mathrm{~km}$ circular polar orbit. One of the experiments to fly on this mission will be the SARA (Sub-keV Atom Reflecting Analyzer) instrument. The SARA experiment is a joint collaborative research program among India, Sweden, Japan, and Switzerland for the exploration of the Moon. In this paper we describe the SARA experiment that will use LENA (low energy neutral atom) as a diagnostic tool to do remote sensing in the lunar environment, and discuss the scientific objectives of this experiment.

\section{$1.1 L E N A$}

The low energy neutral atoms (LENAs) are defined as energetic neutral atoms that have energies in the range $10 \mathrm{eV}$ to about a few $\mathrm{keV}$. LENAs are not bound by the gravitation force of a planetary body, because they move with a velocity much greater than the escape velocity of $2.38 \mathrm{kms}^{-1}$, and being

Keywords. Moon, Moon-solar wind interaction; lunar elemental composition; lunar magnetic anomalies; Energetic Neutral Atom (ENA); lunar mission. 


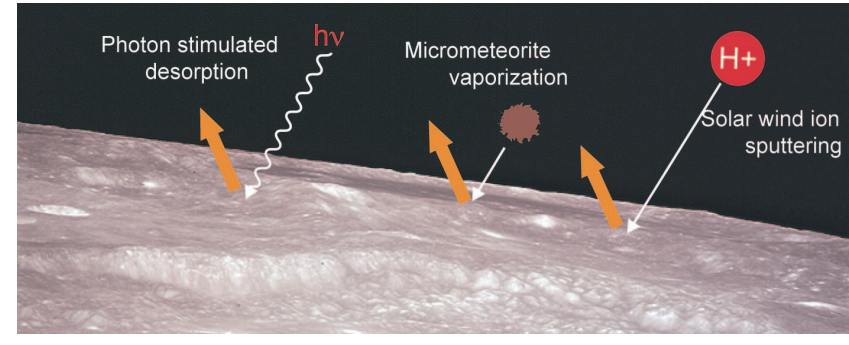

Figure 1. Schematic diagram showing various mechanisms that can produce neutral atoms (LENA) on the Moon.

neutral, are not affected by the electromagnetic forces. The LENAs thus propagate straight, similar to a photon, and can be used to diagnose and image target and parent population and to learn about processes involved. The SARA experiment will sense LENAs in the energy range of 10 to $3000 \mathrm{eV}$.

\subsection{Production and imaging of LENA on the Moon}

Since the Moon does not possess a large-scale intrinsic magnetic field, and thus has no global magnetosphere, as well as is lacking in any extended atmosphere, the LENAs in the Moon environment can result from the following processes (cf. figure 1):

- surface sputtering by precipitating ions,

- incident solar photon stimulated desorption (PSD), also called 'photon sputtering',

- micrometeorite impact vaporization, and

- solar wind backscattering.

The micrometeorite impact produces atoms having a temperature in the range $2000-5000 \mathrm{~K}$ (Eichorn 1978). Assuming a Maxwellian distribution with an average gas temperature of $4000 \mathrm{~K}$ (Wurz and Lammer 2003), the energy of LENA would be $0.34 \mathrm{eV}$, which is too low an energy to be detected by a LENA instrument. Atoms generated by PSD have an energy spectrum $f(E) \propto E^{-(\beta+1)}$, where $\beta$ varies from 0.25 to 0.7 (Johnson et al 2002). Thus, atoms produced by PSD have very low energies (mostly close to the binding energy of atoms, which is in the range $\sim 2-4 \mathrm{eV}$ ), and hence will not be detected by a LENA instrument whose low-energy cutoff is $10 \mathrm{eV}$. However, surface sputtering by precipitating ions can produce LENAs in the energy range of our interest (Futaana et al 2005).

The Moon moves around the Earth at a mean orbital distance of $60 \mathrm{R}_{\mathrm{E}}\left(\mathrm{R}_{\mathrm{E}}\right.$ is Earth radius), and is mainly located in the undisturbed solar wind, except when the lunar phase angle is $<30^{\circ}$ (i.e., when the Moon lies in the Earth's magnetotail).
The sputtered flux is proportional to the precipitating particle flux and it's energy (Sigmund 1969; Johnson 1990). Since the flux and energy of solar wind plasma is generally higher than the magnetotail plasma, the dominant sputtering population is the solar wind.

Sputtering is the process whereby a particle is ejected from its lattice site as a result of the kinetic energy deposited in the lattice by an incident ion (Behrisch and Wittmaack 1991). In the solar wind case, an incident proton initiates a collision cascade in the chemical lattice as it penetrates and comes to rest in a grain of lunar regolith. The cascade can eject an atom of a species by physical sputtering if it reaches the top layers of the grain with an energy exceeding the binding energy of the species to the grain (Sigmund 1969; Johnson 1990). The physical sputtering yield in a multi-component material like the lunar regolith is a function of the binding energy, the relative concentration of the species, and the incident particle energy (Betz and Wehner 1983).

The major elements present in the lunar surface are $\mathrm{O}, \mathrm{Si}, \mathrm{Al}, \mathrm{Mg}, \mathrm{Ca}, \mathrm{Fe}, \mathrm{Na}$, and $\mathrm{Mg}$; other elements have abundance much lower than $1 \%$. The most abundant species is $\mathrm{O}$ at $60 \%$ level (abundance in number), followed by $\mathrm{Si}$ at $16 \%$. For a typical solar wind condition, density $7 \mathrm{~cm}^{-3}$ and velocity $450 \mathrm{~m} \mathrm{~s}^{-1}$, the calculated LENA fluxes, in the energy range of $10-100 \mathrm{eV}$ are of the order of $10^{5} \mathrm{~cm}^{-2} \mathrm{sr}^{-1} \mathrm{~s}^{-1}$ for $\mathrm{O}$ atoms, about $10^{4}-10^{5} \mathrm{~cm}^{-2} \mathrm{sr}^{-1} \mathrm{~s}^{-1}$ for $\mathrm{Ca}, \mathrm{Si}, \mathrm{Al}, \mathrm{Mg}$ atoms, and $\sim 10^{3}-10^{4} \mathrm{~cm}^{-2} \mathrm{sr}^{-1} \mathrm{~s}^{-1}$ for $\mathrm{Fe}$ and $\mathrm{Na}$ atoms (Futaana et al 2005). The energy spectrum of the LENA is presented in Futaana et al (2005).

Since the energy needed to escape lunar gravity for iron $(\mathrm{Fe})$ is $1.62 \mathrm{eV}\left(\mathrm{E}_{\text {esc }}\right.$ at Moon $=0.029 \mathrm{eV} /$ amu), all the dominant LENA produced on the Moon will propagate straight, unaffected by lunar gravity. If remote sensing of LENAs is done from an orbital height of $100-200 \mathrm{~km}$, as in the case of Chandrayaan-1 mission, all the produced LENAs will be detected because this altitude is much smaller than the photoionization scale length. For example a $10 \mathrm{eV}$ potassium $(\mathrm{K})$, which has the highest photoionization rate of all the elements contained in lunar regolith has a photoionization scale length of $\sim 10^{5} \mathrm{~km}$. Hence, the LENAs can be used to perform remote sensing in the Moon environment.

\section{The SARA experiment}

The SARA instrument consists of three major subsystems: a SARA LENA sensor $(\mathrm{CENA}=$ Chandrayaan LENA), a SARA solar wind monitor (SWIM), and a SARA Digital Processing Unit 


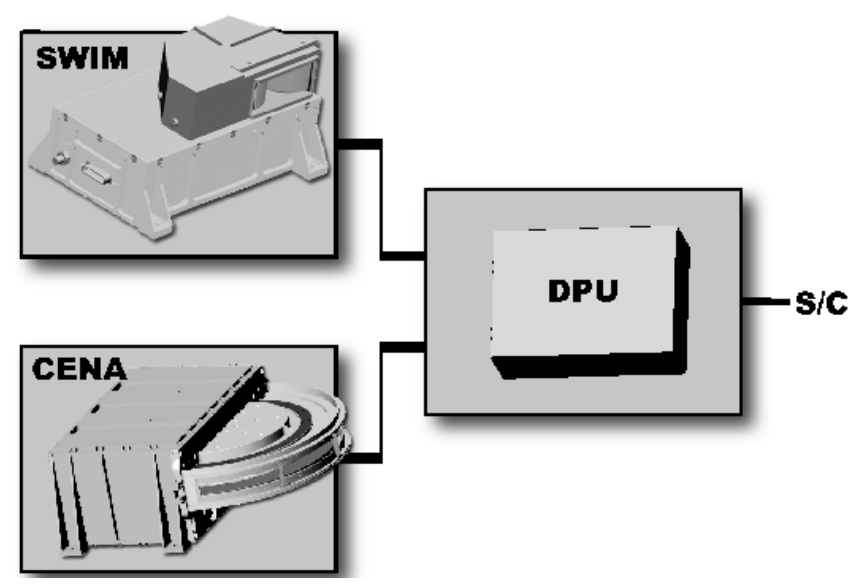

Figure 2. Block diagram of the SARA experiment. S/C means spacecraft. The pictures of CENA, SWIM, and DPU are only schematics and are not drawn according to their real sizes.

(DPU). Mechanically CENA and SWIM are two different units that are connected to DPU by cables, and only the DPU interfaces with the spacecraft (cf. figure 2). The total mass of the SARA experiment is about $3.5 \mathrm{~kg}$ and requires a power of about $4 \mathrm{~W}$. All the commands to CENA and SWIM will be routed through the DPU. The functions of DPU include CENA and SWIM sensor data acquisition, data compression and packaging, house keeping and communicating with the spacecraft. In the following sections we describe the method of working of CENA and SWIM sensors and their important characteristics.

\subsection{The CENA sensor}

The CENA (Chandrayaan LENA) sensor comprises a unique design optimized for:

- very low resource budgets,

- high geometrical factor and high mass resolution,

- wide mass range,

- low energy particle detection $\left(\mathrm{E}_{\min }=10 \mathrm{eV}\right)$,

- high signal-to-noise ratio.

The CENA consists of four subsystems, namely,

(1) a charged-particle removal system (ion deflector),

(2) a conversion surface,

(3) an energy analysis system (which also performs efficient photon rejection), and

(4) a detection system that provides the mass (velocity) analysis.

Figure 3 shows a schematic view of the CENA (Kazama et al 2005).

\subsection{Principle of LENA detection by CENA}

Particles enter the CENA sensor through an electrostatic charged particle deflector, which sweeps away ambient charged particles with energies up to $\sim 15 \mathrm{keV}$ by a static electric field. The incoming neutral particles are then positively ionized by being reflected from a conversion surface and then passed through an electrostatic analyzer of a specific 'wave' shape that effectively blocks photons (cf. figure 4). The electrostatic analyzer also provides crude energy analysis. The 'wave' design of the electrostatic analyzer is similar to the design used in the MTOF (solar wind Mass TimeOf-Flight) sensor of the CELIAS (Charge, Element, and Isotope Analysis System) instrument (Hovestadt et al 1995) on the SOHO spacecraft, which provides a UV photon rejection factor of $2 \times 10^{-8}$.

Since lunar regolith contains elements in significant amounts up to mass $56(\mathrm{Fe})$, CENA must be capable of measuring these elements, i.e., up to Fe. This means no carbon foils can be used in the time-of-flight (TOF) section. To measure the particle velocity (mass) we use the particle reflection technique developed and utilized in the Neutral Particle Detector (NPD) of the ASPERA-3 and ASPERA-4 experiments aboard Mars Express and Venus Express missions of European Space Agency (Barabash et al 2004). After emerging from the electrostatic wave analyzer, the ion is post accelerated by a voltage of about $3 \mathrm{keV}$ and impacts on a START surface under a grazing angle of about $15^{\circ}$. During the impact, kinetic secondary electrons are emitted from the START surface. The incoming particle is reflected towards the STOP MCPs (Micro-channel Plates) where it is detected and produces a STOP pulse. The secondary electrons from the START surfaces are guided to the START MCPs and produce a START pulse. The START and STOP timing gives the TOF, and thus the particle velocity. The mass of the particle is determined by combining the TOF measurement and the energy derived from the electrostatic analyzer.

The START MCP provides the two-dimensional position (in radius and azimuth) and the timing of the particle reflected on the START surface. The radial position allows accurate determination of the TOF length, and the azimuthal position provides the azimuthal angle of the incoming particle. We have performed a full particle-tracing calculation to maximize the performance of the instrument; all major mass groups (H, O, Na, K, and $\mathrm{Fe}$ ) can be resolved by the CENA sensor (Kazama et al 2005). The instantaneous field-of-view (FOV) of CENA is $17^{\circ} \times 160^{\circ}$ and it is always in the nadir viewing geometry (cf. figure 5). It has seven channels, each 


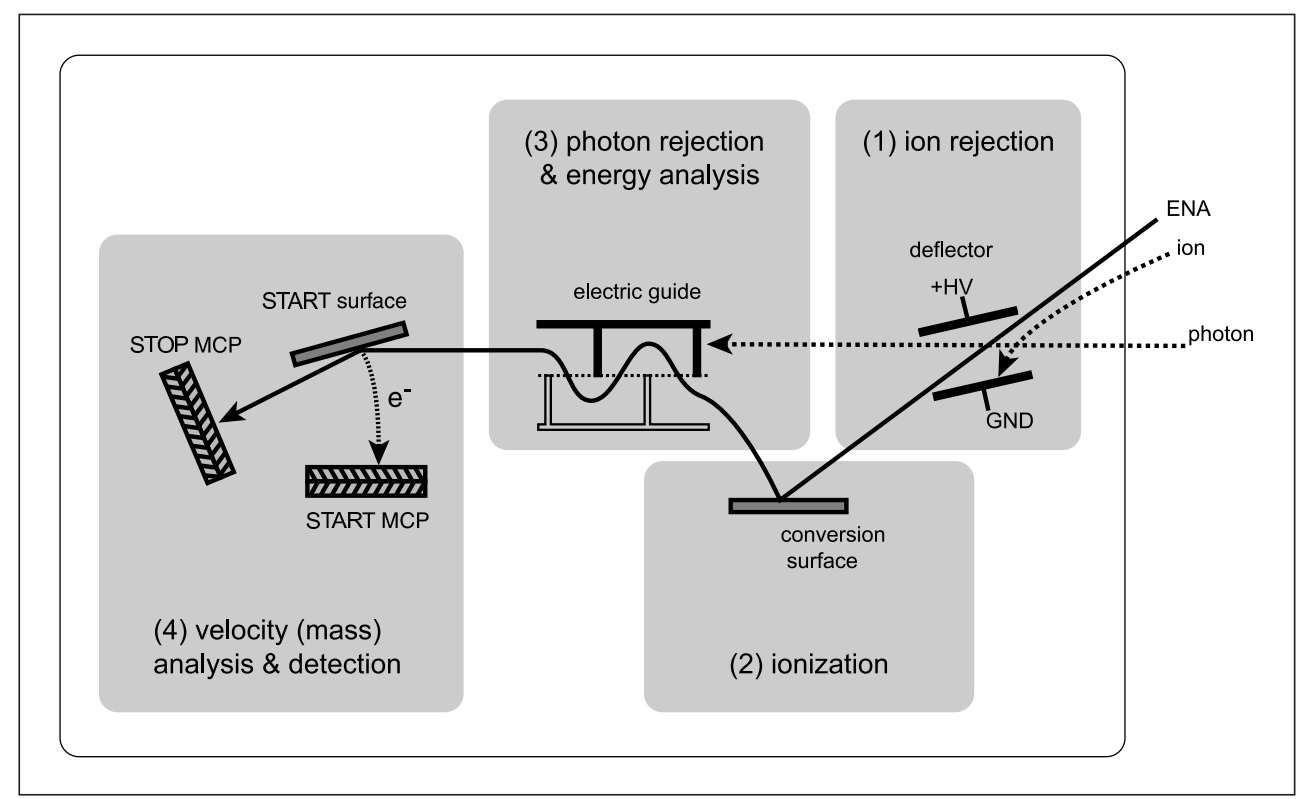

Figure 3. Schematic flow diagram of the CENA instrument showing the principle of the LENA detection.
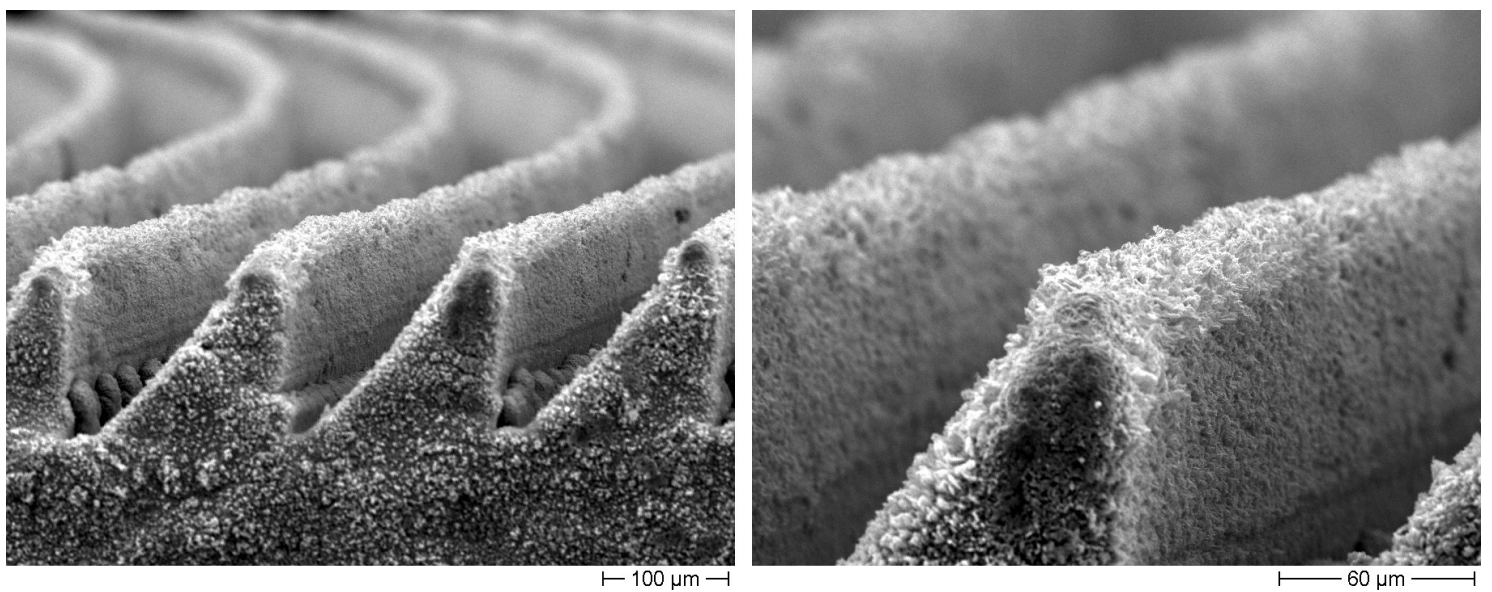

Figure 4. The left image is a close-up view of the serations for the UV suppression elements in CENA, and the right is a zoom of the left image. The height of the serations is $0.177 \mathrm{~mm}$ and the pitch is $0.147 \mathrm{~mm}$. The serated surfaces were coated with copper sulfide to increase the UV suppression.

with a FWHM FOV of $9^{\circ} \times 25^{\circ}$. More details of the CENA are given in Kazama et al (2005). Table 1 summarizes the characteristics of the CENA sensor. Figure 6 provides view of the FOV of the CENA on the lunar surface.

\subsection{The SWIM sensor}

The flux of LENAs produced by the sputtering process is a function of solar wind flux impacting the lunar surface. Therefore, it is required to monitor the precipitating flux of the solar wind on the Moon. The solar wind monitor (SWIM) will provide measurements of energy-per-charge and number density of precipitating ions causing sputtering, which are important inputs in making a quantitative interpretation of the LENA measurements in the lunar environment.

Besides monitoring of the solar wind, there are several scientific topics related to ions around the Moon that SWIM could investigate. For example, the lunar-originated ions reflected from the surface can give important data about the lunar surface composition (Elphic et al 1991). Directly connected with the LENA investigations of the possible mini-magnetospheres on the Moon (see sections 3.3 and 3.4) would be the measurements of ion composition in and around these minimagnetospheres. Such measurements could provide valuable information regarding the interaction 


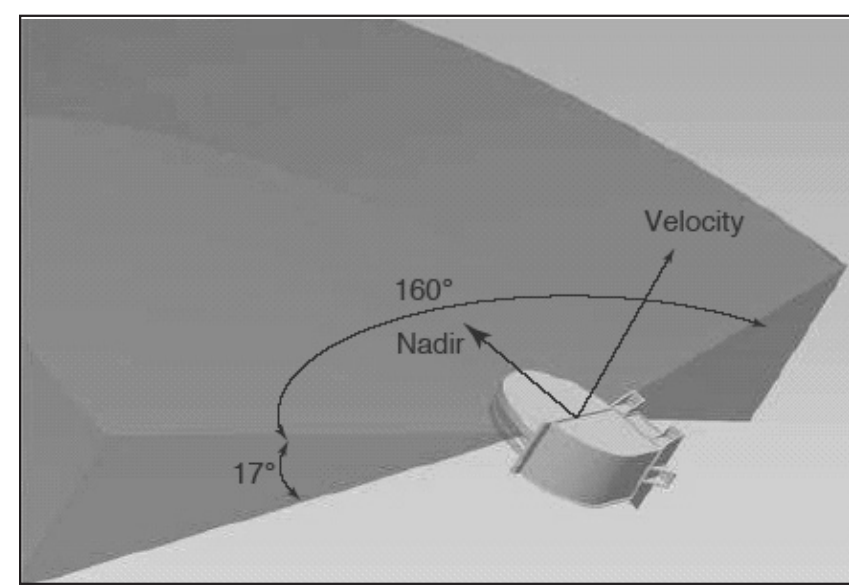

Figure 5. Field of view of the CENA with respect to spacecraft velocity vector and look angle (nadir). CENA is always looking towards the Moon, with its FOV centered around nadir.

Table 1. Characteristics of the SARA CENA sensor.

\begin{tabular}{lc}
\hline \multicolumn{1}{c}{ Parameter } & Value \\
\hline Energy range & $10 \mathrm{eV}-3.3 \mathrm{keV}$ \\
Energy resolution, $\Delta E / E$ & $50 \%$ \\
Mass resolution & $\mathrm{H}, \mathrm{O}, \mathrm{Na}-\mathrm{Mg}$-group, K-Ca- \\
& group, Fe \\
Pure geometrical factor & $10^{-2} \mathrm{~cm}^{2} \mathrm{sr} \mathrm{eV} / \mathrm{eV}$ sector \\
Total efficiency & $\sim 1 \%$ \\
Angular resolution, FWHM & $9^{\circ}(\mathrm{elevation}) \times 25^{\circ}$ (azimuth) \\
Field of view & $17^{\circ} \times 160^{\circ}$ \\
\hline
\end{tabular}

between the anomalies and the solar wind, as well as studying the existence of ion void regions around the anomalies. Moreover, studying the electromagnetic environment around the terminator with SWIM will provide useful science. The terminator is the boundary for photoelectrons, which cause a complicated environment, and in turn could perturb the solar wind (especially the solar wind protons) in this region. Studying the disturbed solar wind in this region could make it possible to map the potential structures around the terminator.

SWIM is a simple ion mass analyzer optimized to provide monitoring of the precipitating ions using little spacecraft resources. Mechanically SWIM consists of two parts: (1) a sensor, and (2) an electronic board that includes high voltage supply and sensor electronics.

Figure 7 shows a 3 -dimensional picture of the SWIM sensor. The ion flux arrival angle is analyzed by a two $90^{\circ}$ plate electrostatic deflector (scanner) and the energy by a $128^{\circ}$ cylindrical electrostatic analyzer. The ions exiting the energy analyzer are post accelerated up to $1 \mathrm{keV}$ energy and are made to strike a START surface. The ion optic is optimized to minimize the spot on the START surface

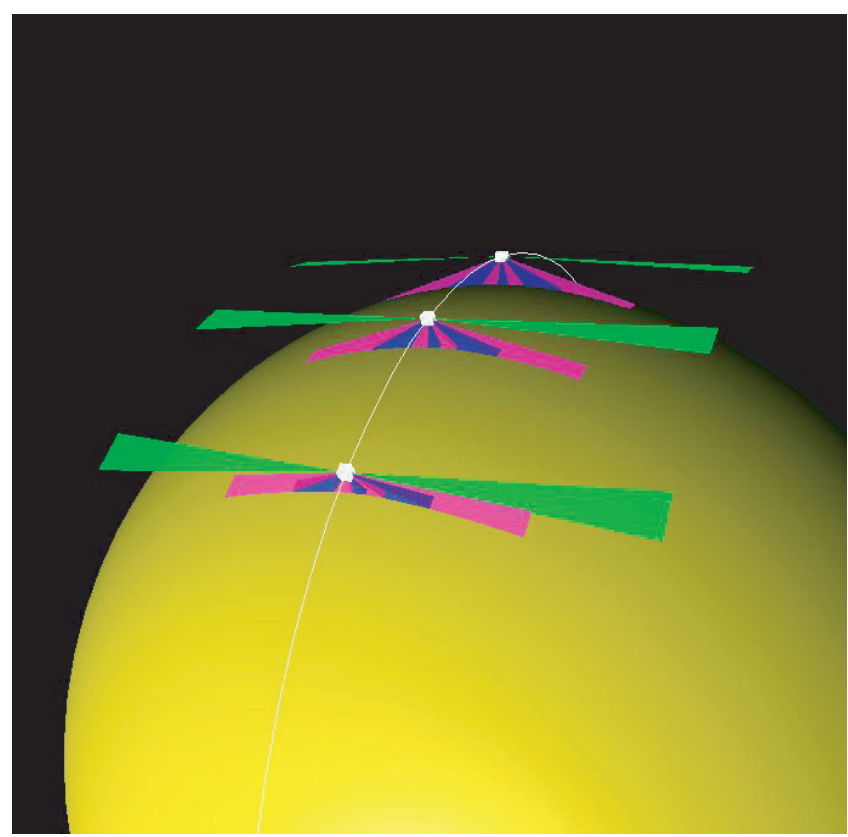

Figure 6. Field of view of the CENA instrument mapped on the lunar surface. The green bands show the full $160^{\circ} \mathrm{FOV}$ of the CENA instrument, while magenta and blue coloured bands show the portion of the FOV which is within the sphere of the lunar surface.

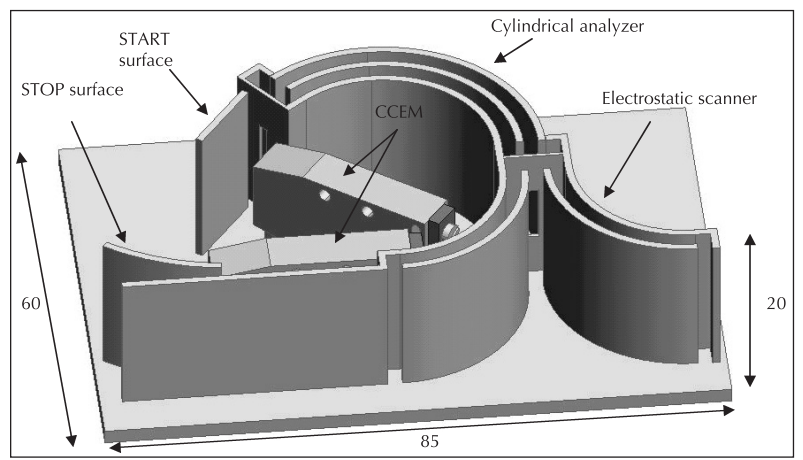

Figure 7. Three-dimensional view of the SARA SWIM instrument. All the major elements of the instrument are labeled. Dimensions are in $\mathrm{mm}$.

and provide a shallow $\left(\sim 15^{\circ}\right)$ incident angle to increase the secondary ion yield and reflection efficiency. The START surface is a specially prepared multilayer surface optimized for

- high secondary electron yield,

- high particle reflection efficiency, and

- large UV photon absorption.

During impact the incident ion produces secondary electrons and it is reflected towards a STOP surface. The generated electrons are collected by a channel electron multiplier and they in turn produce a START pulse. The STOP surface is optimized for the high secondary electron yield and a high UV absorption. Secondary electrons generated 
Table 2. Characteristics of SARA solar wind monitor (SWIM).

\begin{tabular}{ll}
\hline \multicolumn{1}{c}{ Parameter } & \multicolumn{1}{c}{ Value } \\
\hline Energy range & $10 \mathrm{eV}-15 \mathrm{keV}$ \\
Energy resolution, $\Delta E / E$ & $7 \%$ \\
Viewing angle & $9^{\circ} \times 180^{\circ}$ \\
Angular resolution & $9^{\circ} \times 22.5^{\circ}$ \\
Mass resolution & $6 \mathrm{mass}$ groups \\
Energy-elevation cycle & $8 \mathrm{sec}$ \\
Geometric factor, total & $0.14 \mathrm{~cm}^{2} \mathrm{sr} \mathrm{eV}$ \\
\hline
\end{tabular}

from the STOP surface by the impact of the incident particle are collected by the second electron multiplier and this gives the STOP pulse. The time difference between START and STOP events gives the ion velocity and, in combination with the known energy, the mass. The energy analyzer is equipped with a UV trap to increase UV absorption of the system. It is possible to cover an azimuth of $180^{\circ}$ and the energy range from $10 \mathrm{eV}$ to $15 \mathrm{keV}$ by stepping high voltage on the deflector and analyzer.

The sensor envelope is very small, $55 \times 85 \mathrm{~mm}^{2}$. The frame of the instrument, including the deflector and the analyzer grounded structures, is machined from a single block of aluminium. Table 2 sums up the SARA Solar Wind Monitor characteristics.

\section{Science objectives of the SARA}

Imaging of LENAs sputtered from the lunar surface by the SARA will allow us to address the following scientific objectives:

- Moon's surface composition.

- Imaging of permanently and temporally shadowed areas.

- Lunar surface magnetic anomalies and related physics.

In the following we will briefly elaborate on each of the scientific-usages of the SARA experiments mentioned above.

\subsection{Imaging of lunar surface composition}

For tightly bound solids the sputtering yield is proportional to the elastic energy transferred by the incident particle to target atoms near the surface. The fluxes of the sputtered atoms depend on the sputter yield, which is a function of the precipitating flux and the surface composition. Figure 8 shows the precipitating flux of the solar wind in a latitude-longitude map. The precipitating flux is proportional to cosine of the solar zenith angle
(SZA). The white rectangle in the center is the projection of the FOV of the CENA (imager placed at the $100 \mathrm{~km}$ height above the sub-solar point). The relative yield of different elements reflects the surface composition. Figure 9 shows the expected flux of oxygen LENA with energies $>10 \mathrm{eV}$ at the subsolar point and at the terminator. At all channels, the expected oxygen fluxes are well above the threshold level of 500 counts $\mathrm{cm}^{-2} \mathrm{sr}^{-1} \mathrm{~s}^{-1}$ for the CENA sensor. Similarly, for other elements, whose sputtered fluxes are given in section 1.2, the expected flux at the instrument position is above the one-count threshold level. Essentially, this means that all these elements can be detected by the CENA in the lunar environment. The LENA imaging will thus provide maps of the sputtered elements, which can be converted to coarse surface composition maps with a typical spatial resolution similar to the spacecraft height above ground.

\subsection{Imaging temporally and permanently shadowed region}

The Moon's spin axis is nearly perpendicular to the ecliptic $\left(1.5^{\circ}\right.$ tilt $)$, causing locations near the pole to experience extreme solar illumination conditions. Within $20^{\circ}$ of the lunar poles there are significant amounts $(\sim 25 \%)$ of permanently shadowed areas inside craters, whose total area could be $\sim 6000 \mathrm{~km}^{2}$ (Bussey et al 2003). The temperature in these permanently shadowed 'cold traps' regions in the craters is low enough that water ice is stable for billions of years in these areas (Vasavada et al 1999). Recent simulations suggest that the cold trap regions can retain about $6 \%$ of the hydrogen material delivered to these regions by the solar wind after space weathering processes effects are taken into account (Crider and Vondrak 2003). Moreover, the location of the hydrogen-rich areas detected by Lunar Prospector correlates well with the locations of permanent shadow (Feldman et al 2001). This shows a strong temperature dependence on the retention of hydrogen at the lunar poles, interpreted by Feldman et al (2001) as evidence that the hydrogen is in the form of water ice. Though sunlight does not reach these regions, the solar wind particles can penetrate into these shadowed regions, because of their gyro-radius effect (Futaana et al 2005), and cause sputtering, thereby exposing the surface material present there. LENA images from such areas will provide information about surface composition of these regions that is not possible by conventional reflectance or X-ray fluorescence studies.

LENA imaging may also be possible even behind the optical terminator as indicated by the study of Futaana et al (2005). Since the solar wind is a supersonic flow and the Moon is an obstacle to 


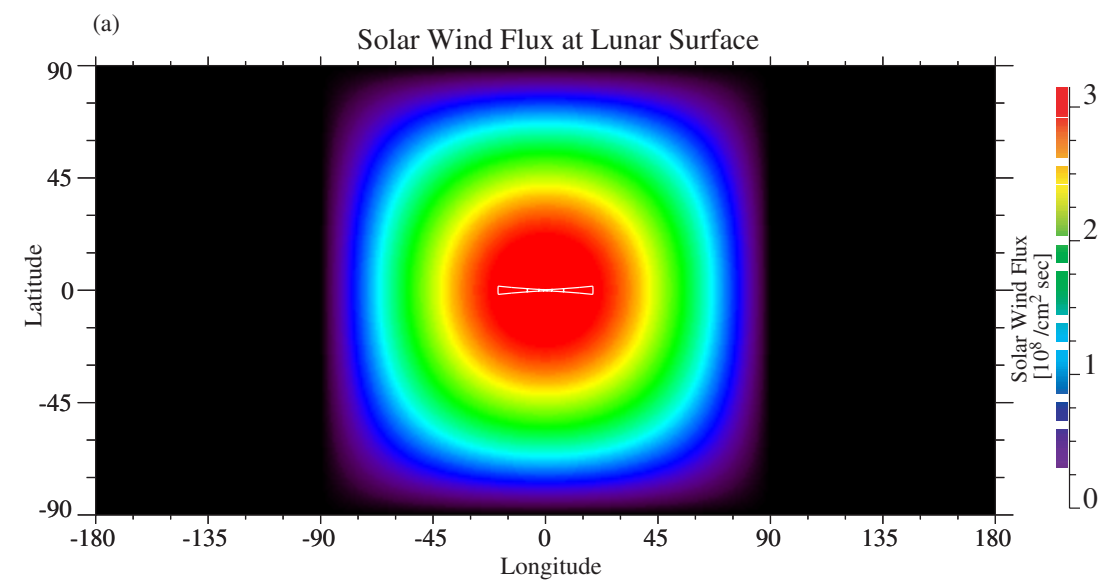

(b)

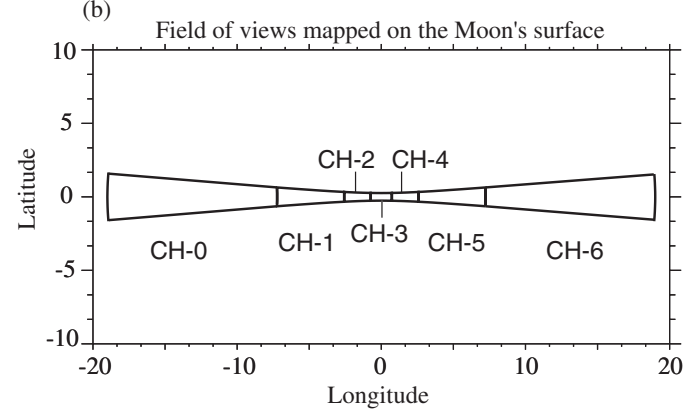

Figure 8. Upper panel: Distribution of the precipitating solar wind flux at the lunar surface. The typical values of the solar wind velocity and density are taken as $450 \mathrm{kms}^{-1}$ and $7 \mathrm{~cm}^{-3}$, respectively. White rectangles show the field of view of each channel $(\mathrm{CH})$ mapped on the lunar surface. The center of the longitude and latitude is the subsolar point. Lower panel: Close-up view of the subsolar region showing the FOV of each channel mapped on the lunar surface.

this flow, a region void of solar wind is generated behind the Moon. The solar wind flowing behind the obstacle is accelerated by a pressure gradient created by its thermal velocity, enabling its penetration into the void region. The solar wind electrons tend to penetrate into the void region faster than the protons, because of their much lower mass, and the resulting charge separation will cause an electric field that accelerates the protons and decelerates the foregoing electrons, like in the case of ambipolar diffusion. The temporal and spatial scale of the charge separation is related to the electron plasma frequency (typically $10 \mathrm{kHz}$ ) and the Debye length (typically $20 \mathrm{~m}$ ). Treating the plasma phenomena on a lunar global-scale, which is typically $1000 \mathrm{~km}$, such a microscopic view can be simplified with the MHD approximation. The MHD theory for plasma diffusion into the void region has been developed (see review by Samir et al 1983), and applied to the conditions at the Moon by Futaana et al (2005). The results of Futaana et al (2005) suggest that for typical solar wind conditions up to $4 \%$ of the upstream solar wind flux can reach areas as far as $10^{\circ}$ beyond the terminator; while in slow solar wind velocity conditions $\sim 8 \%$ of solar wind can reach $20^{\circ}$ beyond terminator.

\subsection{Study of the magnetic anomaly regions}

Although it was previously believed that the lunar magnetic field was too weak to repel solar wind ions, intriguing magnetic anomalies on the lunar surface have been found that can stand off the solar wind, thus creating the smallest known magnetospheres, magnetosheath, and bow shock regions in the solar system (Lin et al 1998). These magnetic anomalies range in magnitude from a few tenths of a nanotesla (nT; $1 \mathrm{nT}=10^{-5} \mathrm{G}$ ) to hundreds of $\mathrm{nT}$, and ranging in size from a few $\mathrm{km}$ to several hundred $\mathrm{km}$, and are present over much of the lunar surface (Hood et al 1979, 2001; Halekas et al 2001). The magnetized regions located near the Imbrium and Serenitatis antipodes are 1200 and $700 \mathrm{~km}$, respectively, with a field of $\sim 300 \mathrm{nT}$ for the entire region. These anomalies are strong enough to form small dipole magnetic fields, creating 'mini-magnetospheres', around $100 \mathrm{~km}$ in diameter locally due to interaction with the solar wind (Lin et al 1998). The solar wind flux cannot reach the surface in these areas and no sputtering of surface material will occur. Thus the expected LENA flux will be extremely low from locations of minimagnetospheres. Simulations of the variation of the solar wind precipitation flux as seen from CENA 


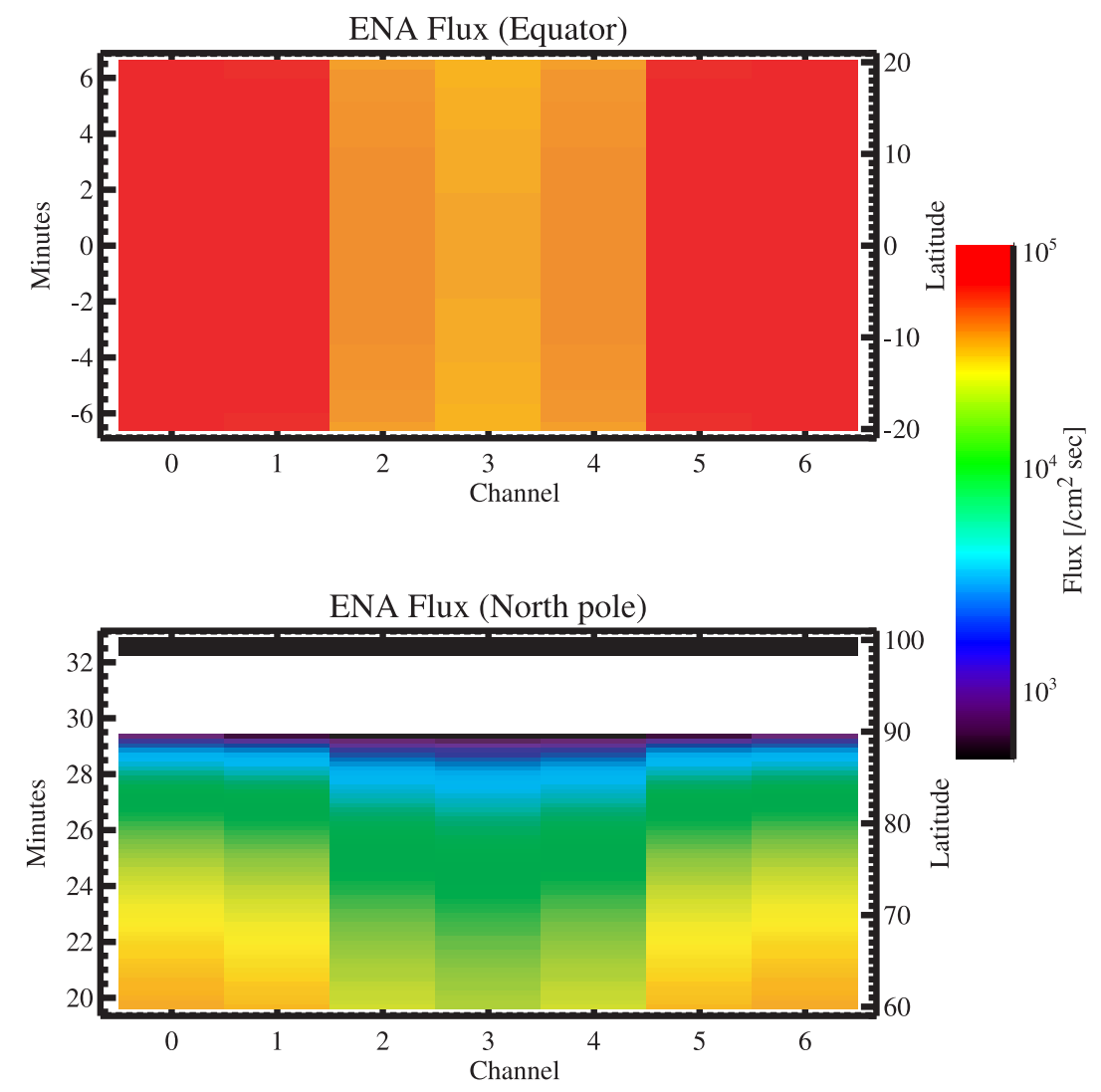

Figure 9. Computed image of oxygen LENA flux as observed by CENA at the subsolar point (upper panel) and at the terminator (lower panel). Each channel $(\mathrm{CH})$ has an intrinsic FOV of $9^{\circ} \times 25^{\circ}$. The 7 channels correspond to a total longitudinal coverage of about $20^{\circ}$ on either side of the center point (see lower panel of figure 8).

(cf. figure 10) reveal that the magnetic anomalies are clearly seen in the LENA images (Futaana et al 2005), which will allow us to study these miniature magnetospheres and hopefully provide global maps of such magnetic anomalies.

\subsection{Mapping of the magnetic anomaly: Implications for space physics}

A mini-magnetosphere is an expected but still unproven concept. Its size is close to the proton gyro-radius for the Moon conditions and this effect gives rise to completely new physical phenomena. Lin et al (1998) presented observational evidence from Lunar Prospector that the interaction between lunar magnetic anomalies and the solar wind could result in the formation of mini-magnetospheres on the Moon. Two-dimensional MHD simulations of the solar wind interaction with the lunar magnetic anomalies made by Harnett and Winglee (2000, 2002) have demonstrated that the crustal magnetic fields can hold off the solar wind and form a "minimagnetosphere'. They concluded that the formation is realized when the field strength is greater than $10 \mathrm{nT}$ at $100 \mathrm{~km}$ above the lunar surface and the solar wind ion density and velocity equal to $10 \mathrm{~cm}^{-3}$ and $400 \mathrm{kms}^{-1}$, respectively. The MHD results also indicated that mini-magnetospheres are much more dynamic than planetary sized magnetospheres. Simulations of solar wind interaction with multiple dipoles on the lunar surface indicate that mini-magnetospheres will form with magnetic field magnitude smaller than the lower limit for a single-dipole (Harnett and Winglee 2003). Observations of non-thermal ions of lunarorigin by Particle Spectrum Analyzer/Ion Spectrum Analyzer onboard Nozomi spacecraft also suggest the formation of mini-magnetospheres (Futaana et al 2003). The mini-magnetospheres should also produce radio emissions (Kuncic and Cairns 2004). The interplanetary magnetic field (IMF) will also affect the formation and shaping of mini-magnetospheres. Flipping of the direction of the IMF from southward to northward can cause the mini-magnetospheres to double their size and change from an elongated shock surface to a round shock surface.

It is thought that most structures resulting from the Moon-solar wind interaction are too small to be detected by in situ observations from an orbit at $100 \mathrm{~km}$. LENA imaging technique is a possible 

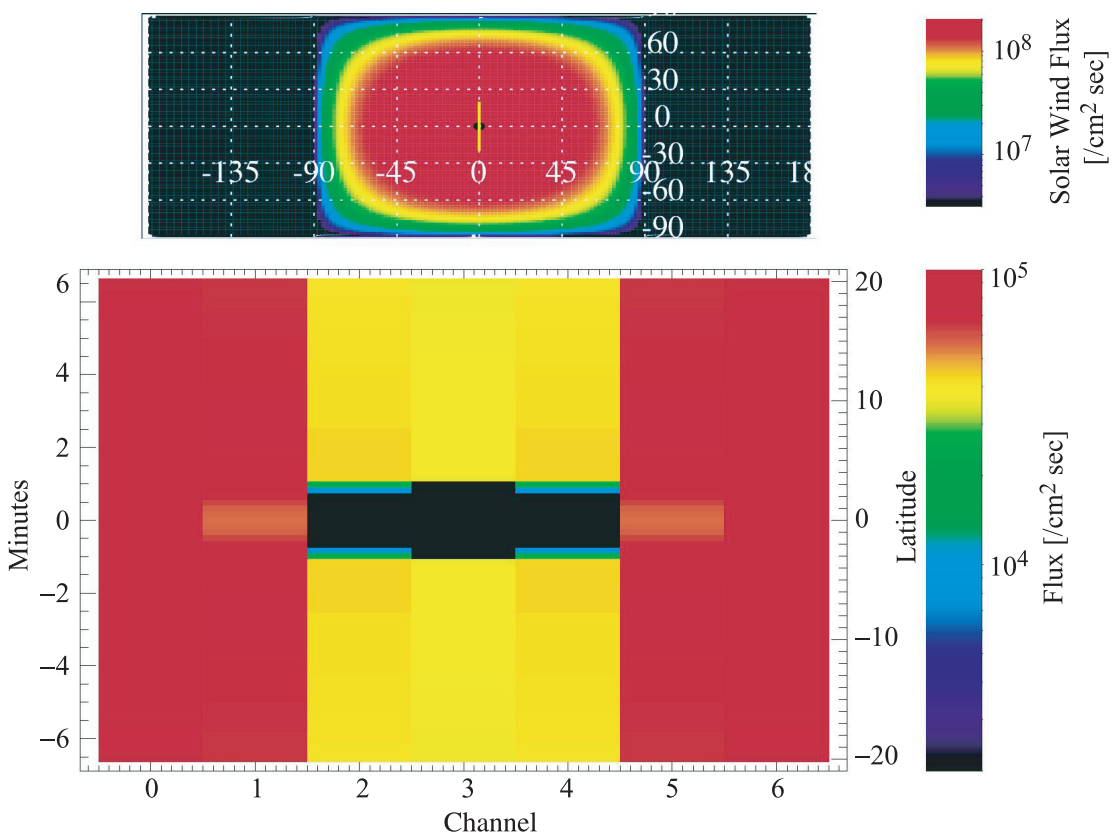

Figure 10. Modeled solar wind flux (upper panel) and images of expected oxygen LENAs for magnetic anomaly located at the subsolar point. Magnetic anomalies are modeled by the disappearance of LENA flux due to the formation of minimagnetosphere. The yellow line shows the spacecraft trajectory.

tool to detect the interaction and to measure the spatial size of mini-magnetospheres. The results of such studies are very important for understanding plasma physics at an ion gyro-radius scale. Moreover, the interaction between the magnetic anomalies and plasma environment changes significantly when the Moon is in the solar wind (note that even for the steady solar wind the precipitation flux on the day and night sides of the Moon are quite different), in the Earth's magnetotail, or in the magnetosheath. These natural variations of the incoming flux can be used to understand formation of the mini-magnetospheres under varying conditions, a unique opportunity not present even for the Earth magnetosphere.

\subsection{Other scientific objectives of the SARA}

In addition to the main objectives discussed above, the SARA instrument can also be used to study:

- Space weathering on the Moon,

- Sources of the exospheric gases and comparative studies of the exospheric gas production at the Moon and Mercury,

- Interplanetary LENAs.

\subsubsection{Studies of space weathering}

Space weathering is a term used to refer to the physical and chemical changes that occur to exposed material on the surface of an airless body. Apart from micrometeoroid bombardment and electromagnetic radiation, solar wind sputtering is an important contributor to the space weathering on the Moon, and all of them are responsible for the formation and maturation of the lunar regolith as a whole. Space weathering is of considerable interest for studies of surfaces by remote sensing because it causes major changes in the optical properties. It is established in laboratory experiments that the proton irradiation of loose powders results in their darkening and reddening (see review by Hapke 2001). High albedo at regions of magnetic anomalies is most probably a manifestation of this effect (Hood et al 2001; Richmond et al 2003, 2005). Inside the magnetic anomalies, the regolith is shielded from the solar wind flux while the surrounding areas receive a higher flux because of the formation of a mini-magnetosphere (Lin et al 1998; Richmond et al 2005). Comparison of the LENA maps with optical or infrared images can directly reveal the quantitative relations between changes in the albedo and the total dose. It is needless to emphasize that an understanding of space weathering is of key importance to the lunar science.

\subsubsection{Study of the Moon's exosphere}

The lunar exosphere is composed of atoms liberated from the lunar surface via interactions with sunlight, the solar wind, and meteorite bombardment. The relative importance of these processes is uncertain, though considerable progress has been 
made in the last decade (Stern 1999; Killen and Ip 1999). One method of distinguishing exospheric source mechanisms is by determining the energy (velocity) distribution of the atoms within the exosphere, since each process results in its own unique velocity distribution of atoms ejected from the surface (see section 1.2). It is established that sputtering contributes significantly to sources of such atoms as $\mathrm{Na}, \mathrm{K}, \mathrm{Al}, \mathrm{Mg}$, Si, Ca (Potter and Morgan 1994; Killen and Ip 1999; Wurz and Lammer 2003).

However, the major problem in quantitative studies of the contribution from sputtering arises from the lack of reliable sputter yields for different species of the mineralogical surface, especially under the influence of space weathering. All the previous studies rely on laboratory measurements made with specially prepared specimens and theoretical studies dealing with simplified assumptions concerning the structure of the sputtered materials. However, the yields strongly depend on the microstructure of the material (solid, porous, loose aggregates, powders, etc.). It is only the direct measurements in space with real planetary surfaces that can establish these vitally important parameters. Absolute measurements of sputtered LENAs with mass resolution combined with knowledge of the solar wind flux and surface composition will be used to derive the sputter yields. These measurements are of significant importance not only for studies of the lunar exosphere but also for that of Mercury and for asteroids studies.

\subsubsection{Studies of the interplanetary neutrals}

The LENA measurements on the Moon will also complement the studies of neutrals in the interplanetary medium, e.g., interstellar helium, the neutral component of the solar wind, and unknown neutral atom beams. The flux of the interstellar helium results from the solar system motion relative to the local interstellar medium (LISM). It has been detected directly by Witte et al (1993; see also Witte 2004). Recently, the LENA experiment on IMAGE made direct measurements of the neutral component in the solar wind (Collier et al 2001). These neutrals are produced due to charge exchange between solar wind ions and the interstellar hydrogen as well as due to the solar wind interaction with the interplanetary dust. However, the exact origin of this component is still being debated. Recently, the IMAGE group reported that there are some indirect indications, coming from different satellites and techniques, that there might exist a third beam of neutrals apart from the interstellar and solar wind particles (Collier et al 2003). Barabash (private communication, 2004) while analysing data from an ENA instrument onboard Mars Express identified some unexplained features, which might be interpreted as the second neutral beam of unknown origin. More advanced measurements are needed for sorting out this issue. The SARA experiment with its $160^{\circ} \mathrm{FOV}$ is capable of observing interplanetary neutrals and thus will be in an advantageous position to study these phenomena. Moreover, since the process of production of interplanetary neutrals is similar to that of the ENA production in planetary magnetospheres (e.g., Henderson et al 1997; Bhardwaj and Gladstone 2000; Moore et al 2000; Brandt et al 2001; Mauk et al 2003), the study of interplanetary ENA will add new dimensions to comparative ENA-planetology.

\section{Relevance of SARA to the Chandrayaan-1 mission}

India's first mission to the Moon, Chandrayaan-1, will carry a set of remote sensing payloads to pursue high resolution mapping of topographic features in 3-D and distribution of various minerals and chemical species covering the entire lunar surface. SARA will complement these measurements by providing coarse maps of elemental distribution over the entire Moon surface including some of the permanently shadowed craters in the outermost layer of regolith. Mass dependent LENA images will also be used to constrain the surface mineralogical composition as derived from the elemental composition maps. SARA will also provide direct maps of the magnetic anomalies capable of withstanding the solar wind dynamic pressure. Studies on lunar exosphere and space weathering effects on lunar surface will also be attempted.

Thus, the observations from SARA will provide the first information on LENA in the lunar environment and will supplement the scientific objectives of Chandrayaan-1. A replica of the SARA CENA sensor is included in the payload of the BepiColombo Mercury Magnetospheric Orbiter. Therefore, conducting the SARA experiment on Chandrayaan-1 opens ample possibilities for doing comparative planetological studies in the nearfuture.

\section{Acknowledgements}

The Swiss contribution to SARA is made possible by the financial support from the Swiss National Science Foundation and by the Swiss PRODEX committee. A part of this research was performed while A Bhardwaj held the NRC Senior Research Associateship at NASA Marshall Space Flight Center. 


\section{References}

Adimurthy V and Ramanan R V 2004 Launch strategy for Indian lunar mission; presented at "International Conference on Exploration and Utilization of the Moon (ICEUM-6)", Nov. 22-26, 2004, Udaipur, India, abstract p. 55.

Barabash S, Lundin R, Andersson H, Gimholt J, Holström M, Norberg O, Yamauchi M, Asamura K, Coates A J, Linder D R, Kataria D O, Curtis C C, Hsieh K C, Sandel B R, Fedorov A, Grigoriev A, Budnik E, Grande M, Carter M, Reading D H, Koskinen H, Kallio E, Riihela P, Säles T, Kozyra J, Krupp N, Livi S, Woch J, Luhmann J, McKenna-Lawlor S, Orsini S, Cerrulli-Irelli R, Mura A, Milillo A, Roelof E, Williams D, Sauvaud J-A, Thocaven J-J, Winningham D, Frahm R, Scherer J, Sharber J, Wurz P and Bochsler P 2004 ASPERA-3: Analyzer of space plasmas and energetic atoms for Mars Express, ESA Special publication ESA-SP-1240, 121-139.

Behrisch R and Wittmaack K 1991 Introduction; In: Sputtering by Particle Bombardment III; (eds) R Behrisch and K Wittmaack (New York: Springer-Verlag) pp. 1-13.

Betz G and Wehner G 1983 Sputtering of multicomponent materials; In: Sputtering by Particle Bombardment II; (ed.) R Behrisch (New York: Springer-Verlag) pp. 11-90.

Bhandari N 2004 Scientific Challenges of Chandrayaan-1: The Indian Lunar Polar Orbiter Mission; Curr. Sci. 86 1489-1498.

Bhardwaj A and Gladstone G R 2000 Auroral emissions of the giant planets; Rev. Geophys. 38 295-353.

Brandt P C, Barabash S, Roelof E C and Chase C J 2001 Energetic neutral atom imaging at low altitudes from the Swedish microsatellite Astrid: Observations at low $(10 \mathrm{keV})$ energies; J. Geophys. Res. 106(A11) $24,663-24,674$.

Bussey D B J, Lucey P G, Steutel D, Robinson M S, Spudis P D and Edwards K D 2003 Permanent shadow in simple craters near the lunar poles; Geophys. Res. Lett. 30(6) 1278, doi:10.1029/2002GL016180.

Collier M R, Moore T E, Ogilvie K W, Chornay D, Keller J W, Boardsen S, Burch J, El Marji B, Fok M-C, Fuselier S A, Ghielmetti A G, Giles B L, Hamilton D C, Peko B L, Quinn J M, Roelof E C, Stephen T M, Wilson G R, Wurz P 2001 Observations of neutral atoms from the solar wind; J. Geophys. Res. 106 24,893-24,906.

Collier M R, Moore T E, Simpson D, Roberts A, Szabo A, Fuselier S, Wurz P, Lee M A, Tsurutani B T 2003 An unexplained $10-40^{\circ}$ shift in the location of some diverse neutral atom data at $1 \mathrm{AU} ; A d v$. Space Res. 34(1) 166-171.

Crider D H and Vondrak R R 2003 Space weathering effects on lunar cold trap deposits; J. Geophys. Res. 108(E7) 5079, doi:10.1029/2002JE002030.

Eichorn G 1978 Heating and vaporization during hypervelocity particle impact; Planet. Space Sci. 26 463-467.

Elphic R C, Funsten III H O, Barraclough B L, McComas D J, Paffett M T, Vaniman D T and Heiken G 1991 Lunar surface composition and solar wind-induced secondary ion mass spectrometry; Geophys. Res. Lett. 18(11) 2165-2168.

Feldman W C, Maurice S, Lawrence D J, Little R C, Lawson S L, Gasnault O, Wiens R C, Barraclough B L, Elphic R C, Prettyman T H, Steinberg J T and Binder A B 2001 Evidence for water ice near the lunar poles; J. Geophys. Res. 106 23,231-23,252.

Futaana Y, Machida S, Saito Y, Matsuoka A and Hayakawa H 2003 Moon-related nonthermal ions observed by Nozomi: Species, sources, and generation mechanisms; J. Geophys. Res. 108(A1) 1025, doi:10.1029/2002JA009366.

Futaana Y, Barabash S, Holmström M and Bhardwaj A 2005 Low energy neutral atoms imaging of the Moon; Planet Space Sci. (in press).

Goswami J N 2005 Chandrayaan-1: Technological and Scientific Challenges, presented at IAA Asia-Pacific regional conference on "Advances in Planetary Exploration", June 26-29, 2005, Bangalore, India.

Halekas J S, Mitchell D L, Lin R P, Frey S, Hood L, Acuña M and Binder A B 2001 Mapping of lunar crustal magnetic fields using Lunar Prospector electron reflectometer data; J. Geophys. Res. 106 27,841-27,852.

Hapke G 2001 Space weathering from Mercury to the asteroid belt; J. Geophys. Res. 106 10,039-10,073.

Harnett E M and Winglee R M 2000 Two-dimensional MHD simulation of the solar wind interaction with magnetic field anomalies on the surface of the moon; J. Geophys. Res. 105 24,997-25,007.

Harnett E M and Winglee R M 2002 2.5-D particle and MHD simulations of mini-magnetospheres at the Moon; J. Geophys. Res. 107(A12) 1421, doi:10.1029/ 2002JA009241.

Harnett E M and Winglee R M 2003 2.5-D fluid simulations of the solar wind interacting with multiple dipoles on the surface of the Moon; J. Geophys. Res. 108(A2) 1088, doi:10.1029/2002JA009617.

Henderson M G, Reeves G D, Spence H E, Sheldon R B, Jorgensen A M, Blake J B and Fennell J F 1997 First energetic neutral atom images from Polar; Geophys. Res. Lett. 24 1167-1170.

Hood L L, Coleman P J Jr and Wilhelms D E 1979 The Moon: Sources of the crustal magnetic anomalies; Science $20453-57$.

Hood L L, Zakharian A, Halekas J, Mitchell D L, Lin R P, Acuña M H and Binder A B 2001 Initial mapping and interpretation of lunar crustal magnetic fields using Lunar Prospector magnetometer data; J. Geophys. Res. $10627,825-27,839$.

Hovestadt D, Hilchenbach M, Bürgi A, Klecker B, Laeverenz P, Scholer M, Grünwaldt H, Axford W I, Livi S, Marsch E, Wilken B, Winterhoff H P, Ipavich F M, Bedini P, Coplan M A, Galvin A B, Gloeckler G, Bochsler P, Balsiger H, Fischer J, Geiss J, Kallenbach R, Wurz P, Reiche K-U, Gliem F, Judge D J, Ogawa H S, Hsieh K C, Möbius E, Lee M A, Managadze G G, Verigin M I and Neugebauer M 1995 CELIAS Charge, Element and Isotope Analysis System for SOHO; Solar Physics 162 441-481.

Johnson R E 1990 Energetic Charged-Particle Interactions with Atmospheres and Surfaces; (New York: SpringerVerlag).

Johnson R E, Leblanc F, Yakshinskiy B V and Madey T E 2002 Energy distributions for desorption of sodium and potassium from ice: The Na/K ratio at Europa; Icarus 156 136-142.

Kazama Y, Barabash S, Bhardwaj A, Asamura K, Futaana Y, Holmström M, Lundin R, Sridharan R and Wurz P 2005 Energetic neutral atom imaging mass spectroscopy of the Moon and Mercury environments; Adv. Space Res., in press, doi:10.1016/j.asr.2005.05.047.

Killen R M and Ip W-H 1999 The surface-bounded atmospheres of Mercury and the Moon; Rev. Geophys. 37 361-406.

Kuncic Z and Cairns I H 2004 Radio emission from minimagnetospheres on the Moon; Geophys. Res. Lett. 31, L11809, doi:10.1029/2004GL020008. 
Lin R P, Mitchell D L, Curtis D W, Anderson K A, Carlson C W, Mc-Fadden J, Acuña M H, Hood L L and Binder A 1998 Lunar surface magnetic fields and their interaction with the solar wind: Results from Lunar Prospector; Science 281 1480-1484.

Mauk B H, Mitchell D H, Krimigis S M, Roelof E C and Paranicas C P 2003 Energetic neutral atoms from a trans-Europa gas torus at Jupiter; Nature $\mathbf{4 2 1}$ 920-922.

Moore T E, Chornay D J, Collier M R, Herrero F A, Johnson J, Johnson M A, Keller J W, Laudadio J F, Lobell J F, Ogilvie K W, Rozmarynowski P, Fuselier S A, Ghielmetti A G, Hertzberg E, Hamilton D C, Lundgren R, Wilson $\mathrm{P}$, Walpole $\mathrm{P}$, Stephen $\mathrm{T} \mathrm{M}$, Peko B L, Van Zyl B, Wurz P, Quinn J M and Wilson G R 2000 The low energy neutral atom imager for IMAGE; Space Sci. Rev. 91(1-2) 155-195.

Potter A E and Morgan T H 1994 Variation of Lunar Sodium Emission Intensity with phase angle; Geophys. Res. Lett. $212263-2266$.

Richmond N C, Hood L L, Halekas J S, Mitchell D L, Lin R P, Acuña M and Binder A B 2003 Correlation of a strong lunar magnetic anomaly with a high-albedo region of the Descartes mountains; Geophys. Res. Lett. 30(7) 1395, doi:10.1029/2003GL016938.

Richmond N C, Hood L L, Mitchell D L, Lin R P, Acuña M and Binder A B 2005 Correlations between magnetic anomalies and surface geology antipodal to lunar impact basins; J. Geophys. Res. 110 E05011, doi:10.1029/2005JE002405.

Samir U, Write K H Jr and Stone N H 1983 The expansion of plasma into a vacuum: basic phenomena and processes and applications to space plasma physics; Rev. Geophys. and Space Phys. 21 1631-1646.

Sigmund P 1969 Theory of sputtering. I. Sputtering yield of amorphous and polycrystalline targets; Phys. Rev. 184 383-416.

Stern S A 1999 The lunar atmosphere: History, status, current problems, and context; Rev. Geophys. 37(4) 453-491.

Thyagarajan K and Annadurai M 2004 Chandrayaan-1: Spacecraft design and technical challenges; paper presented at "International conference on Exploration and Utilization of the Moon (ICEUM-6)", Nov. 22-26, 2004, Udaipur, India, abstract p. 54.

Vasavada A R, Paige D A and Wood S E 1999 Near-surface temperatures on Mercury and the Moon and the stability of polar ice deposits; Icarus 141 179-193.

Witte M, Rosenbauer H, Banaszkiewicz M and Fahr H 1993 The ULYSSES neutral gas experiment: Determination of the velocity and temperature of the interstellar neutral helium; Adv. Space Res. 13(6) 121-130.

Witte M 2004 Kinetic parameters of interstellar neutral helium; Astron. Astrophys. 426 835-844.

Wurz P and Lammer H 2003 Monte-Carlo simulations of Mercury's exosphere; Icarus 164(1) 1-13. 